\title{
The association between rheumatoid arthritis and the HLA antigen DR4
}

\author{
JAN TORE GRAN, GUNNAR HUSBY, AND ERIK THORSBY \\ From Department of Rheumatology, Institute of Clinical Medicine, University of Troms $\emptyset$, Troms $\emptyset$, Norway, \\ and Tissue Typing Laboratory, Rikshospitalet, Oslo, Norway.
}

SUMMARY One hundred and forty-two patients with 'definite' or 'classical' rheumatoid arthritis (RA) were studied for the frequency and possible prognostic significance of HLA DR4. Of these, 122 were seropositive, while 20 were negative for rheumatoid factor (RF) in serum. The HLA antigen DR4 was significantly increased in the seropositive group (65\%) as well as in the seronegative group (55\%) in comparison with a frequency of $27 \%$ in 116 healthy controls. Among seropositive patients a higher frequency of DR4 was found in females (73\%) than in males $(50 \%)$, the difference being statistically significant $(\mathrm{p}<0.01)$. DR4 was more frequent among patients with a family history of RA (74\%) than among patients without affected relatives (57\%). DR4 appeared to be associated with an early onset of RA. No significant differences in general disease activity or functional capacity between DR4-positive and DR4-negative RA patients were found. Patients without the HLA antigen DR4 had a significantly $(p<0.05)$ higher mean titre of RF than those with the antigen.

The first observation of a possible association between the HLA system and rheumatoid arthritis (RA) was made by Astorga and Williams in $1969 .^{1}$ They reported that lymphocytes from some patients with RA failed to respond to each other in mixed lymphocyte culture (MLC) assay but stimulated and responded when mixed with cells from other, unrelated individuals. A shared HLA antigen among patients was the most likely explanation. Several authors then failed to establish an association of RA with HLA A and B antigens. ${ }^{2-4}$ In 1977, however, McMichael et al. ${ }^{5}$ found that the frequency of the HLA antigen D4 was significantly higher in patients with RA (36\%) than in controls $(13 \%)$. The relative risk of getting RA among Dw4 positive persons was calculated to be $3 \cdot 0$. Their findings were later confirmed by Stastny ${ }^{6}$ and Roitt et al. ${ }^{7}$ Furthermore, it has been assumed that patients with positive tests for DR4 are more likely to have severe RA. ${ }^{5}{ }^{7}$ It was also suggested by Khan et al. ${ }^{8}$ that there exists a definite subtype of RA characterised by HLA DR4 positivity, high levels of rheumatoid factor, an aggressive clinical course, and a propensity for familial aggregation of disease. At the 8th International Workshop in

Accepted for publication 29 October 1981.

Correspondence to J. T. Gran, MD, Department of Rheumatology, University Hospital of Troms $\varnothing, 9000$ Troms $\emptyset$, Norway.
Histocompatibility testing ${ }^{9}$ and in the studies of Dobloug et al. ${ }^{10}$ a normal frequency of DR4 in seronegative RA patients was reported.

A preliminary report on HLA DR4 in RA published by our groups ${ }^{11}$ confirmed the observation of increased frequency of HLA DR4 in seropositive RA patients. However, no association between HLA DR4 and an aggressive clinical course could be established. We here report an extended study on the association between HLA DR4 and RA and various disease parameters of this disorder.

\section{Patients and methods}

Patients. 142 patients, 101 females and 41 males, aged $23-82$ years (mean age $56 \cdot 3$ years) with 'definite' or 'classical' rheumatoid arthritis according to the American Rheumatism Association (ARA) criteria $^{12}$ were studied. All patients were admitted to hospital in the Department of Rheumatology, Regionsykehuset, Troms $\varnothing$, Norway, thus presumably representing the most severly diseased patients in our population. All patients were consecutively subjected to tissue typing without any selection. 141 of the patients were Caucasians, while one was of Lappish origin. The average duration of the disease was 
$10 \cdot 1$ years (range 0.5 to 34 years), determined retrospectively by careful questioning of the patients about the start of their arthritic symptoms. All the patients had typical erosive $x$-ray abnormalities characteristic of the disease.

122 patients $(86 \%)$ were positive for rheumatoid factor (RF). Twenty patients $(14 \%)$ had negative reactions when repeatedly tested for RF. Originally the latter group consisted of 40 patients who were observed for a period of 2 years. To be included in the final seronegative group a patient had to fulfil the ARA criteria for RA, and all repeated tests for RF had to be negative. We also required a disease duration of more than 3 years, absence of psoriasis, and no family history of inflammatory bowel disease, psoriasis, or other seronegative rheumatic diseases. At the end of the observation period all patients in this group had $x$-rays of the lower part of the spine and the sacroiliac joints. They were questioned about low back pain and examined for disease of the sacroiliac joints and the spine. Patients with any signs of sacroiliitis were excluded. By these criteria 20 patients were excluded from the original 40 patients with seronegative RA, 9 because roentgenograms showed no signs of peripheral arthritis, 3 converted into seropositivity during the observation period, 2 had roentgenographic evidence of sacroiliitis, 4 developed psoriasis, and 2 were excluded because of psoriasis and ulcerative colitis in their families. Four patients were excluded from the calculations of HLA antigen frequencies because of a familial relationship.

127 patients had received treatment with gold, penicillamine, chloroquine, or systemic corticosteroids. In 15 patients the disease was satisfactorily controlled with salicylates or other nonsteroidal anti-inflammatory drugs.

Assessments. Patient disability and disease severity were assessed by the American Rheumatism Association's functional capacity classification (ARA FC) based on 4 functional classes of RA. ${ }^{13}$ The ARA FC classification was based on a single recording made when the patient was subjected to tissue typing. Disease severity was also assessed in terms of the type of medical treatment required to control disease activity. Drug class I patients managed on salicylates alone; those in class II were treated with nonsteroidal anti-inflammatory drugs; in class III the patients were on gold, chloroquine, or penicillamine; while class IV patients required systemic corticosteroids, cytostatic drugs, or treatment with plasma exchange to control disease activity.

Parameters of general disease activity were: concentrations of haemoglobin and the acute-phase reactants haptoglobin and orosomucoid, thrombocyte count, and the erythrocyte sedimentation rate
(ESR). Duration of morning stiffness was used as a subjective parameter of disease activity. All values were obtained at the time of presentation to tissue typing. The Waaler-Rose test was used for the determination of rheumatoid factor (RF) and the highest individual titre recorded was selected for analysis. A titre of $\geqslant 32$ was required for seropositivity.

HLA typings. Healthy controls included unrelated members of staff of the Tissue Typing Laboratory, blood donors, and relatives of transplant patients previously typed at the Tissue Typing Laboratory (several thousands had been typed for the HLA A, B, $C$ antigens, and 100-200 for the HLA DR antigen). ${ }^{14}$ The HLA typing was performed by a microcytotoxicity technique with 87 highly selected antisera defining $13,21,4$, and 8 antigens of the A, B, C, and DR series respectively by techniques previously described. ${ }^{15}$

Statistics. Student's $t$ test and the chi-square test were used for statistical analyses, and a $p$ value of less than 0.05 was considered to be statistically significant.

\section{Results}

Prevalence of $H L A$ antigens in seronegative and seropositive $R A$. DR4 was found with significantly increased frequency both in the RF-positive group (65\%) and in the RF-negative group (55\%) as compared with a positivity of $27 \%$ found among normal persons (Table 1). The difference between RFpositive and RF-negative RA was not statistically significant. The frequency of DR4 in the original group of $40 \mathrm{RF}$-negative patients was $42.5 \%$, which was also increased, but the increase was not statistically significant when compared with normal controls. To our surprise the DR4-negative patients were shown to have a significantly higher titre of RF than the DR4-positive ones, 759 versus 545 respectively. Among the RF-positive patients the increased frequency of DR4 was most prominent among female patients, namely, $73 \%$ as compared with $50 \%$ among males $(p<0.01)$. The relative risk for persons with DR4 of getting RA was calculated to be $7 \cdot 0$.

The HLA antigen B27 which is known to be

Table 1 Prevalence (\%) of HLA DR4 and HLA B27 in 138 patients with $R A$ and 116 normal controls

\begin{tabular}{|c|c|c|c|c|c|}
\hline & \multicolumn{3}{|c|}{$R F$-positive patients, $n=118$} & \multirow{2}{*}{$\begin{array}{l}R F \text {-negative } \\
\text { patients } \\
n=20\end{array}$} & \multirow{2}{*}{$\begin{array}{l}\text { Normal } \\
\text { controls, } \\
n=116\end{array}$} \\
\hline & Total & Females & Males & & \\
\hline DR4 & $65^{*}$ & $73^{*}$ & $50^{*}$ & $55^{*}$ & 27 \\
\hline B27 & $20^{*}$ & 15 & 24 & 25 & 10 \\
\hline
\end{tabular}

$* \mathrm{p}<0.05$. 
associated with certain seronegative polyarthropathies $^{16}$ was found with increased frequency $(20 \%)$ in RF-positive RA $(p<0.05)$, while the frequency in the RF-negative patients, though similarly increased $(25 \%)$, did not reach statistical significance when compared with the frequency of $10 \%$ among normals (Table 1 ).

$D R 4$ and age at onset of $R A$. The average age at onset of disease for the 142 RA patients was $46 \cdot 2$ years (range 17-81 years). The disease tended to start earlier in females (43.3) than in males $(52.6$ years). The RF positive patients with DR4 got their disease at a lower age ( 44.8 years) than the RFpositive patients without this antigen $(49 \cdot 1$ years). There was no difference in age at onset of RA between DR4-positive and DR4-negative patients in the RF-negative group. The average age at onset was similar in RF-positive and RF-negative RA.

$D R 4$ and functional prognosis of $R A$. The mean ARA FC score of the DR4-positive group $(2 \cdot 2)$ was similar to that of the DR4-negative group $(2 \cdot 3)$. Among the RF-positive patients the ARA FC of the male DR4-positive group $(1 \cdot 7)$ was better than that of the male DR4-negative group $(2 \cdot 2)$, and this difference was statistically significant $(p<0 \cdot 01)$. The mean ARA FC of the RF-negative patients was worse $(2 \cdot 5)$. The RF-negative DR4-positive patients fared worse, though not significantly, than the RFnegative DR4-negative patients. The RF-positive patients with the DR4 antigen had an average disease duration of 10.9 years, which was longer than the duration of 8.8 years in the RF-positive DR4negative group, to some extent reflecting the lower age at onset of RA. The mean age, however, was lower (55.7 years) in the former than in the latter group (57.9 years).

An increasing degree of disability was, as expected, seen with increased disease duration. Age per se is an important factor for the degree of the patients' disability, and with higher age a greater difficulty in performing the different functions included in the ARA FC classification was observed. When further analysed, it appeared that for patients with disease onset after 40 years of age disability increased with both increasing age and disease duration. For patients with onset before the age of 40 , however, neither disease duration nor age seemed to influence the functional prognosis of RA (data not shown).

There was no difference between patients with the DR4 antigen and patients without this antigen with respect to drug classification (data not shown). Similarly there was no significant difference in drug use between the 2 sexes, but a slightly higher use of drugs on average was noted among the DR4-negative males. A comparison of the degree of bony destruction seen on $x$-ray in DR4-positive and DR4-negative patients showed that patients without the antigen had slightly more (but not significant by so) extensive $x$-ray destruction than the DR4-positive patients.

DR4 and general disease activity. There was no significant difference between DR4-positive and DR4-negative patients. The haemoglobin concentration was somewhat lower in DR4-positive than in DR4-negative patients, both in the RF-positive and in the RF-negative group, but the difference was not significant.

The small differences in ESR between DR4positive and negative patients, between RF-positive and RF negative ones, and between males and females did not reach statistical significance. Haptoglobin and orosomucoid levels (not shown) and thrombocyte counts (not shown) showed no significantly deviating values between the different groups. Duration of morning stiffness was the same among DR4-positive and DR4-negative patients. RFnegative patients had slightly more prolonged morning stiffness than the RF-positive patients, the longest duration being found in DR4-positives, but the difference was not significant when compared with DR4-negative patients.

DR4 and serum levels of immunglobulins. All the RA patients had serum levels of the immunglobulin classes, IgG, IgA, and IgM within the normal range. In the RF-positive group the immunglobulin concentrations did not differ significantly among DR4positive and DR4-negative patients. The IgG concentration was similar in RF-positive and RFnegative patients, the concentration of IgM was somewhat higher in the RF-negative group, while the IgA concentration was significantly higher among the RF-positive, $3.3 \mathrm{mg} / \mathrm{ml}$ versus $2.4 \mathrm{mg} / \mathrm{ml}$ respectively (SI: $3 \cdot 3$ and $2 \cdot 4 \mathrm{~g} / \mathrm{l}$ ).

DR4, subcutaneous nodules, and antinuclear factors. Although the difference was not statistically significant, the DR4-negative patients had a higher frequency of subcutaneous nodules and antinuclear factors in the serum (not shown).

$D R 4$ and familial aggregation of $R A$. A positive family history of RA was verified in $39.4 \%$ of the 138 patients. Family members affected by RA were most frequently found in the DR4-positive group (46\%) as compared with a frequency of $28 \%$ in the DR4negative group $(p<0.05) .74 \%$ of the patients with a relative affected by RA carried DR4 as compared with $57 \%$ in patients without affected relatives. It was found that 36 out of 138 patients $(26 \%)$ were neither in possession of the DR4 antigen nor had any family history of RA.

\section{Discussion}

The present study confirms previous reports ${ }^{617}$ of a 
significantly increased frequency of HLA DR4 in patients with RA. The reason why the increase was most prominent among females in our material is unknown, and no similar sex difference was observed among the normal controls.

The frequency of DR4 in seropositive RA (65\%) was found to be only a little higher than in the seronegative group (55\%), and this difference was not statistically significant. Furthermore, the mean titre of RF in the DR4-negative group was indeed found to be significantly higher than that in the DR4-positive group. These findings are supported by those of Panayi and coworkers ${ }^{17}$ and by the study of Engleman et al., ${ }^{18}$ the latter making similar observations on healthy women with positive tests for RF. In contrast, another Norwegian study ${ }^{10}$ found a positive correlation between the presence of DR4 and the production of RF in patients with RA, which also was confirmed by others during the 1980 International Histocompatibility Workshop. ${ }^{9}$ Cross-checking of sera performed blindly revealed no differences between our laboratories with regard to determinations of RF. A more likely explanation for this apparent discrepancy is therefore different criteria for selection of patients in the RF-negative group. In addition, to assure negative sacroiliac joint $x$-rays and the absence of psoriasis, we also ruled out patients with ankylosing spondylitis, psoriatic arthropathy, and inflammatory bowel disease among their first-degree relatives, and required a disease duration of 3 years or more.

Furthermore the prospective aspect of our study, with an observation period of 2 years, permitted us to omit 20 patients with seronegative arthropathies other than RA from the study, thus decreasing the original $40 \mathrm{RF}$-negative patients by $50 \%$. By doing so the frequency of DR4 rose from $42.5 \%$ in the original group to $55 \%$ in the final RF-negative group of RA.

We found higher (not statistically significant) concentrations of IgM in RF-negative RA. This was also at variance with the results of Dobloug et al., ${ }^{10}$ who found the serum concentrations of $\operatorname{IgM}, \operatorname{IgE}$, and $\operatorname{IgA}$ to be significantly higher in seropositive than in seronegative RA. No difference in immunoglobulin concentrations appeared between DR4-positive and negative patients in our study.

In a preliminary report ${ }^{11}$ we questioned the prognostic significance of DR4 in RA, and the present study strongly confirms that no such role of this tissue antigen is evident. Our observations are in agreement with some previous studies ${ }^{19}$ but at variance with others. ${ }^{57}$ Thus McMichael et al. ${ }^{5}$ found that DR4positive patients required more aggressive therapy to control their disease and that they had a slightly more severe disease. It is not clear what parameters other than drug classification the authors used to determine the disease severity. Roitt et al. ${ }^{7}$ found that patients with positive tests for bothDR4 and rheumatoid factor were more likely to have severe disease warranting aggressive therapy, but it is possible that RF was the overriding prognostic factor in their studies. One reason for the discrepancies between our results and those of others ${ }^{58}$ may be that we have used the functional capacity as a main basis for prognosis of RA. We feel uncertain about using the medical therapy needed to control disease activity as an index of the severity of the disease, as it is greatly influenced by the medication policy of the clinicians involved. However, in our studies no difference with respect to drug classification was found between DR4-positive and negative patients.

Our results confirmed previous reports ${ }^{20}$ of an association between presence of DR4 and early onset of RA. In some studies ${ }^{21}$ a late onset on RA has been shown to be associated with a severe prognosis. That appeared to be true also in this study. It cannot be excluded that higher age at onset of RA among the DR4-negative patients compensated for an eventual difference with respect to functional outcome. The 2 groups are comparable with respect to disease duration and age, 2 factors, generally accepted as influencing functional capacity. An interesting observation was that the patient's age and the disease duration influenced the functional capacities only of those patients whose disease started after 40 years of age. It is not clear why the prognosis in our patients with early onset of disease was not influenced by age or disease duration. However, it appears that the disease per se is most important for the functional prognosis when it starts earlier in life.

Our patients represented the most disabled and severely diseased patients requiring admission to hospital for their RA, and among these patients DR4 allowed no discrimination with respect to functional prognosis or general disease activity. To determine more precisely the influence of DR4 on the prognosis of RA, all categories of RA in a given population should be studied prospectively, and such studies are now in progress.

The present study confirms previous observations ${ }^{16}$ that DR4 is particularly likely to be increased in RA patients with a family history of the disease. However, it was interesting that more than $25 \%$ of our patients were negative both with respect to DR4 and a family history of RA, indicating that factors other than the HLA DR4 are related to the aetiology of the disease. A possible explanation is that an antigen closely linked to DR4 is common to all RA patients. Another possibility, yet to be proved, is that RA is a syndrome of multifactorial aetiology. One of the aetiological factors might be DR4 or another but 
related HLA antigen. Only 7 patients (5\%) out of 142 lacked DR4, a positive family history of RA, and $\mathrm{RF}$ in serum. A combination of these 3 factors could therefore be helpful in the diagnosis of RA.

This work was supported by the Norwegian Research Councils for Science and the Humanities, the Norwegian Women's Health Association, and the Norwegian Rheumatism Council.

\section{References}

${ }^{1}$ Astorga G P, Williams R C Jr. Altered reactivity in mixed lymphocyte culture of lymphocytes from patients with rheumatoid arthritis. Arthritis Rheum 1969; 12: 547-54.

${ }^{2}$ Seignalet J, Clot J, Sany J, Serre H. HL-A antigens in rheumatoid arthritis. Vox Sang 1972; 23: 468-71.

${ }^{3}$ Lies R B, Messner R D, Troup G M. Histocompatibility antigens and rheumatoid arthritis. Arthritis Rheum 1972; 15: 524-9.

4 Kueppers F, Brackertz D, Mueller-Eckhardt C H. HL-A antigens in sarcoidosis and rheumatoid arthritis. Lancet 1972; ii: 1425 .

5 McMichael A J, Sasazuki T, McDevitt M O, Payne R O. Increased frequency of HLA-Cw3 and HLA-Dw4 in rheumatoid arthritis. Arthritis Rheum 1977; 20: 1037-42.

6 Stastny P. Association of the B-cell alloantigen DRw4 with rheumatoid arthritis. $N$ Engl J Med 1978; 298: 869-71.

7 Roitt I M, Corbett M, Festenstein H. et al. HLA-DRw4 and prognosis in rheumatoid arthritis. Lancet 1978; ii: 990.

- Khan M A, Kushner, Ballou S P, Braun W E. Familial rheumatoid arthritis and HLA-DRw4. Lancet 1979; ii: 921-2.
- Stastny P. Rheumatoid arthritis. In: Histocompatibility Testing 1980. UCLA Press, 1980: 681-6.

${ }^{10}$ Dobloug J H, Førre $\emptyset$, Kåss E, Thorsby E. HLA antigens and rheumatoid arthritis. Arthritis Rheum 1980; 23: 309-13.

11 Husby G, Gran J T, Østensen M, Johannessen A, Thorsby E. HLA-DRw4 and rheumatoid arthritis. Lancet 1979; i: 549.

12 Ropes M W, Bennet G A, Cobb S, Jacox R, Jessar R A. Diagnostic critieria for rheumatoid arthritis-1959 revision. Bull Rheum Dis 1958; 9: 175-6.

${ }^{13}$ Steinbrocker O, Traeger C H, Batterman R C. Therapeutic criteria for rheumatoid arthritis. JAMA 1949; 140: 659-62.

14 Thorsby E. The human histocompatibility complex HLA: some recent developments. Transplant Proc 1979; 9: 616-23.

15 Albrechtsen D, Bratlie A, Nousiainen H, et al. Serological typing of HLA-D: Predictive value in mixed lymphocyte cultures (MLC). Immunogenetics 1978; 6: 91-100.

16 Brewerton D A. HLA-B 27 and the inheritance of suspectibility to rheumatic disease. Arthritis Rheum 1976; 19: 656-68.

${ }^{17}$ Panayi G S, Wooley P M, Batchelor J R. HLA-DRw4 and rheumatoid arthritis. Lancet 1979; ii: 730.

18 Engleman E G, Sponzilli E E, Batey M E, Ramcharan S, McDevitt H O. Mixed lymphocyte reaction in healthy women with rheumatoid factor. Arthritis Rheum 1978; 21: 690-3.

19 Thomsen M, Morling N, Snorrason E, Sveijgaard A, Sørensen S F. HLA-Dw4 and rheumatoid arthritis. Tissue Antigens 1979; 13: $56-60$.

${ }^{20}$ Bodmer W F, Batchelor J R, Bodmer J G, Festenstein M, Morris P J. Histocompatibility Testing 1977. Copenhagen: Munksgaard, 1978: 218-9.

${ }^{21}$ Fleming A, Crown J M, Corbett M. Prognostic value of early features in rheumatoid disease. $\mathrm{Br}$ Med J 1976; i: 1243-5.

\section{Book review}

Equipment for the Disabled: Wheelchairs. 5th Edn. Wheelchairs. Ed. E. R. Wilshere. Pp. 103. No price given. Manton (Westminster) Ltd. Brighton. 1982.

This book is the newest of a well-known series and has its merits. It addresses itself succintly to the problem (or should I say equipment) and deals logically, clearly, and briefly with the individual chairs, which are arranged in generic groups. There are also sections on cushions and support systems and excellent general guidelines which should be read by all prescribers. Drawings are clear, if small. For once the salesman's line, 'you cannot afford to be without it' is true. All physicians who prescribe wheelchairs of any sort should have it by them. 